\title{
Gradually increased dyslipidemia in human immunodeficiency virus infected male patients with tenofovir plus lamivudine plus efavirenz primary treatment: a 3-year follow-up study
}

dafeng liu ( $\sim$ ldf312@126.com )

the Public and Health Clinic Centre of Chengdu https://orcid.org/0000-0002-6792-641X

Bennan Zhao

the Public and Health Clinic Centre of Chengdu

Xinyi Zhang

Sichuan University West China Clinical Medical College

Fengjiao Gao

the Public and Health Clinic Centre of Chengdu

Jun Kang

the Public and Health Clinic Centre of Chengdu

Xue Zheng

the Public and Health Clinic Centre of Chengdu

Lijuan Lan

the Public and Health Clinic Centre of Chengdu

Yinsheng He

the Public and Health Clinic Centre of Chengdu

Shenghua He

the Public and Health Clinic Centre of Chengdu

Research article

Keywords: human immunodeficiency virus (HIV), highly active antiretroviral therapy (HAART), lipid metabolism parameter, uric acid, dynamic change, long-term

Posted Date: August 13th, 2020

DOl: https://doi.org/10.21203/rs.3.rs-55587/v1

License: (c) (i) This work is licensed under a Creative Commons Attribution 4.0 International License.

Read Full License 


\section{Abstract}

Introduction

Since the start of highly active antiretroviral therapy (HAART) with TDF plus 3TC plus EFV, the long-term dynamic characteristics of lipid and purine metabolism in patients infected with human immunodeficiency virus (HIV) was unclear and worth studying.

Methods

A prospective follow-up cohort study was the way. Sixty-one treatment-naive HIV infected male patients were divided into three groups based on the baseline CD $4+T$ cell count $(26,12,23$ cases in $<200$, from 200 to $350,>350$ three groups, respectively). Lipid and purine metabolism parameters of those patients within 144 weeks were analyzed.

Result

TG, TC, LDL-C and HDL-c level all gradually increased within 144 weeks, but statistical significances of TC level and HDL-c level were only found ( $F=4.214,5.518, P=0.001,0.000$,respectively). Moreover the percentage of hypercholesterolemia, hyper LDL cholesterolemia and hypertriglyceridemia all gradually increased, low HDL cholesterolemia gradually decreased, but there was only obvious difference of the latter $\left(X^{2}=16.105, P=0.0007\right)$. Furthermore the lower the baseline $C D 4+T$ lymphocyte counts, the higher TG level, the lower TC level, LDL-c level and HDL-c level, but only significant difference of LDL-c level between three groups at baseline was found $(F=3.256, P=0.0457)$. Although UA level and the percentages of hyperuricemia gradually increased within 144 weeks, but there was no significant difference between different follow-up time points groups and between three CD4 + T cell count groups (all Pख0.05).

Conclusions

These findings provide a reference for clinicians to monitor lipid metabolism parameters closely during long-term HAART with TDF plus 3TC plus EFV regimen.

\section{Introduction}

In recent years, immune-deficiency virus (HIV) infected and acquired immune deficiency syndrome (AIDS) patients has sharply increased. By the end of 2019 all over the world about 38 million people lived with HIV and 33 million people died of HIV-related diseases[1], at the end of October 2019 nationwide in China a total of 958,000 patients[2,3] lived with HIV,at the end of September 2018 a total of 262,000 patients died of HIV-related diseases in China[2, 3].

The most effective treatment for AIDS is antiretroviral therapy which can prolong life expectancy and improve life quality $[4,5]$. When $\mathrm{CD} 4+\mathrm{T}$ cell count reaches more than $350 / \mathrm{mm}^{3}$ and the viral load reaches undetectable levels within the first year of starting treatment AIDS patients are predicted to have a normal 
life expectancy[4, 5].The cumulative survival rates of AIDS patients have obviously increased[6-8]. As of 2017, 20.9 million HIV infected patients have received antiretroviral treatment all over the world. But metabolic abnormalities, cardiovascular risk factors, and osteoporosis have become important factors affecting the prognosis and life quality of AIDS patients[9-12].

HIV virus infection itself and HAART treatment drugs can cause dyslipidemia. But as a first-line highly active antiretroviral therapy (HAART) program launched since the National Twelfth Five-Year Plan in China, Tenofovir (TDF) plus lamivudine (3TC)plus efavirenz (EFV) regimen has less effect on lipid metabolism, there are few reports about it in the literature. Our previous study has shown that newly diagnosed AIDS male patients had decreased total cholesterol (TC) level, uric acid (UA) level[13, 14], highdensity lipoprotein cholesterol (HDL-c) level and increased triglyceride (TG) level, especially those with CD $4+$ count $<200 / u 1$, this dyslipidemia and decreased UA level gradually returned to normal after 4 weeks since initial HAART with TDF plus 3TC plus EFV regimen[13, 14]. But the long-term dynamic characteristicsoflipid and purinemetabolism in those patients was unclear and worth being identifiedin this study.

\section{Patients And Methods}

\section{Study Population}

A prospective cohort study was conducted on sixty-one male patients with HIV infected and treatmentnaive in the Public and Health Clinic Centre of Chengdu from October 1, 2012 to December 31, 2017.Among them the first 50 cases have been involved in the literature[13-15]. Study was approved by the hospital ethics committee of the Public and Health Clinic Centre of Chengdu. All patients gave written informed consent.

The inclusion criteria were as follows: Age from 18 to 65 years old; Gender is not limited; HIV-1 antibody positive by enzyme-linked immune-sorbent assay test, and confirm by Western blotting;CD4 + T cell count $₫ 500 u L$ within 30 days before enrollment; Sign informed consent voluntarily and guarantee to receive follow-up; No plan to move away from current address during the trial; subjects have not received any antiretroviral therapy before the trial.

The following exclusion criteria were used in this study: patients with acute infections; with opportunistic infections or AIDS-related malignant tumors at the time of enrollment; or an opportunistic infection occurred within 3 months before enrollment, and the condition is still unstable within 2 weeks before enrollment; the following results were detected during screening: hemoglobin $<9 \mathrm{~g} / \mathrm{dL}$, white blood cell count $<2000 /$ LL, neutrophilscount $<1000 /$ uL, platelet count $<75000 /$ LL, serum creatinine $>1.5$-fold upper limit of the normal value (ULN), aspartate aminotransferase/alanine aminotransferase/alkaline phosphatase $>3$-fold ULN, total bilirubin $>2$-fold ULN, serum creatine phosphokinase $>2$-fold ULN;creatinine clearance rate $<60 \mathrm{~mL} / \mathrm{min}$;women during pregnancy and lactation; current drug users; 
with severe mental and neurological diseases; with a history of alcoholism who cannot be terminated; severe digestive tract ulcers.

The diagnostic criteria of the diseases were as follows: AIDS, dyslipidemia and hyperacidemia diagnostic criteria[16-18].

The participants were divided in three groups according to the baseline CD4 T cell counts $(26,12,23$ cases in $<200$, from 200 to 350 and $>350$ cells/ul three groups, respectively).

\section{Detection of Laboratory indicators}

The subjects were fasted overnight for at least 12 hours. At 8:00 am the next day venous blood of those patients was drawn for total cholesterol (TC), low density lipoprotein cholesterol (LDL-C), high density lipoprotein cholesterol (HDL-c), triglyceride (TG), uric acid (UA), HIV viral nucleic acid (HIVRNA), T lymphocyte subsets.

TC, TG, HDL-C, LDL-C and UA were measured by enzymatic method of automatic biochemical analyzer, the kit was purchased from Zhejiang Eastern European Biological Products Company. HIVRNA was detected by fluorescent quantitative PCR; T lymphocyte subsets (including CD3 + count, CD 4 + count, CD8 + count, $\mathrm{CD} 3+\%, \mathrm{CD} 4+\%, \mathrm{CD} 8+\%)$ were measured by flow cytometry using Beckman flowcytometer.

The follow-up time point was at $0,4,8,12,24,36,48,60,72,84,96,108,120,132,144$ weeks since patients accepting HAART with TDF plus $3 T C$ plus EFV regimen. UA was measured at each follow-up time point, TC, LDL-C, HDL-C and TG were detected at 0, 24, 48, 96, 120, 144 weeks.

Databases were established according to the needs of the research by two researchers simultaneously collecting and entering. About $30 \%$ of the data was randomly selected by the researchers to ensure data integrity, authenticity, and accuracy.

\section{Patient and Public Involvement}

Patients and public was involved in the development of the research question or in the design of the study. Patients will receive oral and written information about this trial, however, they will not be involved in the recruitment and conduct of the study. Besides, the burden of the intervention will be assessed by patients themselves. After signing an informed consent by the participants, they will be assessed for eligibility and data collection will begin. Dissemination of the general results (no personal data) will be made on demand.

\section{Statistical Method}

The Statistical Package for the Social Sciences software version 17.0 (IBM Inc., Armonk, NY, the USA) and GraphPad Prism 8 (GraphPad) software were used for statistical analysis. TC level, LDL-c level, HDL-C level, TG level and UA level had a normal distribution, and statistical analysis was conducted directly. Non-normally distributed HIVRNA level was performed naturallogarithmic trans-formation before statistical analysis. The measurement data were expressed as $x \pm$ SD for metrological data, rate or 
percentage for enumeration data. ONE ANOVA was used to compare metabolism parameters from baseline to 144 weeks and a paired t-test was used to compare metabolism parameters between baseline and some follow-up time point. Kruskal-Wallis $\mathrm{H}(\mathrm{K})$ test for $\mathrm{K}$ independent samples was used to compare the percentage of dyslipidemia and hyperuricemia from baseline to 144 weeks. Mann-Whitney test for two independent samples was used to compare the percentage of dyslipidemia and hyperuricemia between baseline and some follow-up time point. ONE ANOVA was used to compare metabolism parameters between three different CD $4+T$ cell count groups at same time point. TWO ANOVA was used to compare metabolism parameters between three different CD4 $+\mathrm{T}$ cell count groups from baseline to 144 weeks. A p value $<0.05$ was considered statistically significant.

\section{Results}

\section{Baseline conditions}

Sixty-one treatment-naive HIV infected male patients in the Public and Health Clinic Centre of Chengdu from October 1, 2012 to Decmenber 31, 2017, were divided into three groups according to the baseline CD4 + T cell count $(26,12,23$ cases in $<200$, from 200 to $350,>350$ cell/ul three groups, respectively). There were 42 cases of homosexual infection, 13 cases of heterosexual infection and 5 cases of two types of homosexual and heterosexual infection. The general information, baseline immunity and virological indicators, lipid metabolism parameters of 61 cases were shown in Table 1. 
Table 1

Baseline information and of HIV infected male patients $(n=61)$

\begin{tabular}{|c|c|c|}
\hline variable & $\chi \pm S D$ or cases(\%) & range \\
\hline age(years) & $32.05 \pm 8.38$ & $20 \bigotimes 58$ \\
\hline gender(male,\%) & $61(100 \%)$ & \\
\hline infection duration(months) & $11.16 \pm 1.19$ & $1 \otimes 86$ \\
\hline \multicolumn{3}{|l|}{ T lymphocyte subsets } \\
\hline CD3 + count (cells/ul) & $1433.98 \pm 595.35$ & $470 \sim 3074$ \\
\hline CD3 + CD $4+$ count (cells/ul) & $313.87 \pm 118.473$ & $54 \sim 499$ \\
\hline CD3 + CD $4+$ percentage $(\%)$ & $19.78 \pm 6.83$ & $1.40 \sim 43.40$ \\
\hline $\mathrm{CD} 3+\mathrm{CD} 8+$ count $(\mathrm{cells} / \mathrm{ul})$ & $1119.70 \pm 605.0$ & $360 \sim 2456$ \\
\hline CD3 + CD 8 + percentage (\%) & $69.97 \pm 13.80$ & $36.13 \sim 97.20$ \\
\hline *Virus load of HIVRNA & $41772.77 \pm 10.38$ & $895.00 \sim 505987.00$ \\
\hline \multicolumn{3}{|l|}{ metabolic parameters } \\
\hline $\mathrm{TG}(\mathrm{mmol} / \mathrm{L})$ & $1.68 \pm 1.23$ & $0.39 \sim 16.81$ \\
\hline $\mathrm{TC}(\mathrm{mmol} / \mathrm{L})$ & $4.20 \pm 0.72$ & $2.38 \sim 6.09$ \\
\hline LDL-c (mmol/L) & $2.61 \pm 0.68$ & $0.92 \sim 4.71$ \\
\hline $\mathrm{HDL}-\mathrm{c}(\mathrm{mmol} / \mathrm{L})$ & $1.12 \pm 0.24$ & $0.57 \sim 1.77$ \\
\hline $\mathrm{UA}(\mu \mathrm{mol} / \mathrm{L})$ & $310.72 \pm 68.65$ & $143 \sim 506$ \\
\hline
\end{tabular}

Effectiveness of HAARTwith TDF plus 3TC plus EFV regimen

In 61 patients the average CD $4+T$ cell count (Fig. 1A) gradually increase from 319.80cell/ul at baseline to $464.85 \mathrm{cell} / \mathrm{ul}$ at 96 weeks after HAART, and the average viral load (Fig. 1B) decreased rapidly from $49846.32 \mathrm{IU} / \mathrm{ml}$ at baseline to reaching undetectable levels by the high-precision detection method at 72 weeks, and the percentage of viral load reaching undetectable levels (Fig. 1C) was from $21.31 \%$ at 12 weeks to $100.00 \%$ at 72 weeks.

Long-term dynamic changes of lipid and purine metabolism parameters after after treatment with TDF + $3 T C+E F V$ 
TC level, LDL-c level, HDL-c level, TG level and UA level (Fig. 2A, 2B, 2C, 2D and 4A) all gradually increased along with prolonged HAART, but the increasing amplitude was small, only statistical significance of TC level (Fig. 2A) and HDL-c level (Fig. 2C) (0, 24, 48, 96, 120, 144 weeks: 4.20vs. 4.13, 4.43, 4.60, 4.44, $4.67 \mathrm{mmol} / \mathrm{L} ; 1.12 v s .1 .19,1.32,1.36,1.23,1.30 \mathrm{mmol} / \mathrm{L} ; F=4.382,6.033, P=0.0007,0.000$, respectively) was found. Compared with baseline, there were significant differences of TC level at 96 and 144 weeks (Fig. 2A), HDL-c level at 48, 96 and 144 weeks (Fig. 2C) (4.20vs. $4.60 \mathrm{mmol} / \mathrm{L}, 4.20 \mathrm{vs} .4 .67 \mathrm{mmol} / \mathrm{L}$; 1.12 vs. $1.32 \mathrm{mmol} / \mathrm{L}, 1.12 \mathrm{vs} .1 .36 \mathrm{mmol} / \mathrm{L}, 1.12 \mathrm{vs} .1 .30 \mathrm{mmol} / \mathrm{L} ; \mathrm{t}=3.155,2.175,3.610,5.189,2.473, \mathrm{P}=$ $0.002,0.0329,0.0004,0.0001,0.0157$, respectively). Comparison between different time points and compared with baseline, no significant difference of LDL-c level (Fig. 2B), TG level (Fig. 2D) and UA level (Fig. 4A) was found ( all $P>0.05$ ).

Simultaneously the percentage of hypercholesterolemia (Fig. 3A), hyper low density lipoprotein cholesterolemia (Fig. 3B) and hypertriglyceridemia (Fig. 3D) all gradually increased, but there was no obvious difference (24.59vs. 29.51, 24.59, 21.31, 25.00, 50.00\%; 9.84vs. 8.20, 8.20, 18.03, 25.00, 21.43\%; $8.20 v s .8 .20,9.84,18.05,10.00,21.43 \%, \chi^{2}=5.351,7.966,5.408, P=0.375,0.158,0.368$, respectively). On the countrary, the percentage of low high density lipoprotein cholesterolemia (Fig. $3 \mathrm{C}$ ) gradually decreased along with prolonged HAART, significant difference was found from baseline to 144 weeks (Fig. 3C) $\left(36.07 v\right.$ v. 18.03, 16.39, 9.84, 20.00, 7.14\%, $\left.\chi^{2}=16.105, P=0.0007\right)$, and at $24,48,96,144$ weeks compared with at baseline (Fig. 3C) (36.07vs. 18.03, 16.39, 9.84, 20.00, 7.14\%, 36.07vs. 18.03\%, 36.07vs. $16.39 \%, 36.07 v s .9 .84 \%, 36.07 v s .7 .14 \% ; Z=-2.233,-2.460,-3.431,-2.102, P=0.026,0.014,0.001,0.036$, respectively). The percentage of hyperucicemia (Fig. 4B) slightly decreased with the extension of the treatment time of HAART, no obvious difference was found $(P>0.05)$.

Effect of baseline CD4 + T cell count on lipid and purinemetabolic parameters after treatment with TDF + $3 T C+E F V$

The lower the CD4 + T cell count at baseline, the higher the TG level (Fig. 5D), and the lower the TC level (Fig. 5A), LDL-c level (Fig. 5B), HDL-c level (Fig. 5C) and UA level (Fig. 6), and the changes were maintained throughout the follow-up period after HAART treatment. However, there was no statistical significance from baseline to 96 weeks between three different CD4 + T cell count groups ( all $P>0.05$ ). Only the difference of LDL-c level (Fig. 5B) at baseline between three different CD $4+T$ cell count groups was found (2.22vs.2.70, $2.67 \mathrm{mmol} / \mathrm{L}, \mathrm{F}=3.256, \mathrm{P}=0.0457$ ). TC level (Fig. 5A) and HDL-c level (Fig. 5C) all gradually increased along with prolonged HAART regardless of the CD4 $+\mathrm{T}$ cell count at baseline.

\section{Discussion}

Currently, most of the literatures focus on the proportion of abnormal lipid metabolism in patients treated with HAART. As reported in the literature, the incidence of dyslipidemia in HIV/AIDS patients is quite common in Asia, especially in some southeast Asian countries, such as $34.93 \%$ in Thailand[19], more than 76\% in Tanzania[20], 82.3\% in southern Ethiopia[21] and 20-100\% in India[22-25]. It has also been reported that $10-60 \%$ of patients receiving HAART treatment have hypercholesterolemia[8, 26-29], 20- 
70\% hypertriglyceridemia[8, 9, 29, 30], 35.1\% were hyper LDL cholesterolemia[29], and 20-68.5\% low high density lipoprotein cholesterolemia[9, 29-32].No report on long-term dynamic changes of specific firstline HAART regimen, especially TDF + 3TC + EFV on lipid and purine metabolism have been reported.

In this prospective cohort study we aimed at identifying the long-term dynamic characteristics of lipid and purine metabolism and its influencing factors after HAART with TDF + 3TC + EFV. To our knowledge, this prospective 3-year follow-up cohort study was the first to report the long-term dynamic effects of TDF + $3 \mathrm{TC}+\mathrm{EFV}$ regimen and baseline CD4 + T cell count on lipid and purine metabolic parameters in HIVinfected primary treatment male patients. The results showed that in patients treated with TDF + 3TC + EFV regimen for 3 years, TC level, LDL-c level and HDL-c level gradually increased, especially TC level and HDL-c level, TG level first gradually decreased and then gradually increased, moreover the percentage of hypercholesterolemia, hyper LDL cholesterolemia and hypertriglyceridemia all gradually increased, while the percentage of low HDL cholesterolemia gradually decreased. It shows that in the early stage of HAART treatment disordered lipid metabolism was improved, espectially low HDL cholesterolemia. But along with prolonged HAART, the proportion of hyperlipidemia and hypertriglyceridemia gradually increased, espectially hyper LDL cholesterolemia and hypertriglyceridemia after 48 weeks, hypercholesterolemia after 120 weeks, which were from $9.84 \%, 8.2 \%, 24.59 \%$ at baseline to $21.43 \%$, $21.43 \%, 50.00 \%$ at 144 weeks, respectively.

This study was a cohort study with a follow-up time of 144 weeks. The proportion of patients with abnormal lipid metabolism during the whole study period was less than that reported in the literature. The reasons may be related to the younger age of patients in this cohort, the duration of follow-up was not too long, and the TDF + 3TC + EFV regimen had little effect on metabolism. It is still necessary to expand the sample size, increase the number of female cases, extend the follow-up time and increase other HAART regimens for further discussion.

Previous study found that high CD4 + cell count was a risk factor for hypertriglyceridemia, while less than 200 copies $/ \mathrm{mm}^{3} \mathrm{CD} 4+$ cell count increased the risk of hypercholesterolemia[29]. Regardless of whether the initial treatment regimen or based on D4T or not, the risk of hyperlipidemia in HIV/AIDS patients aged 50 and above is significantly higher than that in young HIV/AIDS patients aged under 40[33]. In this prospective 3-year follow-up cohort study the long-term effect of baseline CD $4+T$ lymphocyte count on lipid parameters was that the lower the baseline CD $4+T$ lymphocyte count, not only the higher TG level, but also the lower TC level, LDL-c level and HDL-c level.

Literature reported that of patients lived with HIV for mean duration 17.4 years, $35.6 \%$ had ASCVD, of those without ASCVD 53-86\% had intermediate or moderate-to-high 10-year ASCVD risk scores, the cardiovascular risk factors including HIV, 31.9\% low high-density lipoprotein cholesterol, $79.3 \%$ needed to receive statin therapy[34].

Comparison between different time points within 144 weeks and compared with baseline, no significant difference of UA level was found. Although the lower the CD4 + T cell count at baseline, the higher the UA 
level, and the changes were maintained throughout the follow-up period after HAART treatment, but there was also no statistical significance from baseline to 144 weeks between three different CD $4+T$ cell count groups. That is to say that TDF $+3 T C+E F V$ regimen and $C D 4+T$ cell count at baseline had no long-term dynamic effects on purine metabolism.

The present study had some limitations. The sample size was small, and this was a single-center, cohort study which only involved TDF + 3TC + EFV regimen. A further multicenter, more HAART regimens involved, large sample randomized controlled trial is needed.

\section{Conclusions}

These findings provide a reference for clinicians to monitor TG, TC, LDL-C, HDL-c closely during long-term HAART with TDF plus 3TC plus EFV regimen.

\section{Declarations}

\section{Ethics approval and consent to participate}

The study was approved by the ethics committee of the Public and Health Clinic Centre of Chengdu (PJK2012-26-01).A written informed consent was waived by the Ethics Commission of the designated hospital because of emerging infectious diseases.

\section{Consent for publication}

Not applicable.

\section{Competing interests}

The authors declare that they have no conflicts ofinterest to this study.

\section{Funding}

This research was supported by the Twelfth Five-Year Project on Tackling Key Problems of National Science and Technology (2012ZX10001-003), the Sichuan Province Health Commission (130430;17PJ070), Chengdu Municipal Health Commission (2019079).

\section{Authors' contributions}

Concept and design: Dafeng Liu, Bennan Zhao, Xinyi Zhang, Feng jiao Gao, Jun Kang,Xue Zheng,Lijuan Lan, Yinsheng He; Data acquisition:Dafeng Liu, Bennan Zhao, Xinyi Zhang, Feng jiao Gao, Jun Kang,Xue Zheng,Lijuan Lan; data analysis and interpretation: Dafeng Liu, Bennan Zhao, Xinyi Zhang; Drafting the manuscript: Dafeng Liu, Bennan Zhao, Xinyi Zhang; administrative, technical, or material support: Dafeng Liu, Bennan Zhao, Xinyi Zhang; study supervision:Shenghua He. 
Acknowledgments

Thanks to Dr. Lin Cai, Ruifeng Zhou, Yin Wang (the Public and Health Clinic Centre of Chengdu,one ward of infectious disease department).

\section{Conflicts of interest}

The authors declare that they have no conflicts ofinterest to this study.

\section{ORCID}

Dafeng Liu'iDhttps://orcid.org/0000-0002-6792-641X

\section{Data availability statement}

All data, models, or code generated or used during the study are available from the corresponding author by request:Dafeng Liu, E-mail: Idf312@126.com

\section{References}

1. World Health Organization. HIV/AIDS. https://www.who.int/zh/ news-room/fact-sheets/detail/hivaids.

2. Disease Control and Prevention Bureau of National Health Commission of the People's Republic of China. New progress was made in the prevention and treatment of AIDS in China in 2019. http://www.nhc.gov.cn/jkj/s3586/201911/c2388ce70bdd404ea6dfcd886591784d.shtml.

3. Huajing Information Network. In 2019, the number of AIDS cases, deaths and the status of anti-AIDS drugs in China are still facing huge challenges. https://www.huaon.com/story/469227.

4. Samji H, Cescon A, Hogg RS, et al. Closing the gap: increases in life expectancy among treated HIVpositive individuals in the United States and Canada. PLoS One. 2013;8:e81355.

5. May MT, Gompels M, Delpech V, et al. Impact on life expectancy of HIV-1 positive individuals of CD4 + cell count and viral loadresponse. to antiretroviral therapyAIDS. 2014;28:1193-202.

6. Brown TT, Li X, Cole SR, et al. Antiretroviral therapy and the prevalence and incidence of diabetes mellitus in the multicenter AIDS cohort study. Arch Int ern Med. 2005;165:1179-84.

7. Loulergue P, Cal lard P, Bon nard P, et al. Hepatic steatosis as an emerging cause of cirrhosis in HIVinfected patients. J Acquir Immune Defic Syndr. 2007;45:365.

8. Riddle TM, Kuhel DG, Woollett LA, et al. HIV protease inhibitor induces fatty acid and sterol biosynthesis in liver and adipose tissues due to the accumulation of activated sterol regulatory element binding proteins in the nucleus. J Biol Chem. 2001;276:37514-9.

9. Samaras K, Wand H, Law M, et al. Prevalence of metabolic syndrome in HIV-infected patients receiving highlyactive antiretroviral therapy using International Diabetes Federation and Adult treatment Panel III criteria. Diabetes Care. 2007;30:113-5. 
10. Stanley TL, Grinspoon SK. Body compositiom and metabolic changes in HIV-infected patients. J Infect Dis. 2012;205:383-90.

11. Adelzon A, Paula M, Schechter SuelyHTuboi, et al. Continuous Increase of Cardiovascular Diseases, Diabetes, and Non-HIV Related Cancers as Causes of Death in HIV-Infected Individuals in Brazil: An Analysis of Nationwide Data.Plos2014;9:1-5.

12. Muyanja D, Muzoora C, Muyingo A, Muyindike W, Siedner MJ. High Prevalence of Metabolic Syndrome and Cardiovascular Disease Risk Among People with HIV on Stable ART in Southwestern Uganda. AIDS Patient Care STDS. 2016;30:4-10.

13. Lan LJ, Liu DF, Chen Z, He SH, Liu YL, et al. The glucolipid metabolism characteristics of newly diagnosed male patients with acquired immune deficiency syndrome.Chin. J Exp Clin Infect Dis (Electronic Edition). 2016;10(3):280-4.

14. Liu Dafeng $H$, Shenghua $Z$, Ruifeng, et al. Dynamic changes of early metabolic parameters in newly treated HIV patients with TDF + 3TC + EFV. AIDS STDs in China. 2014;20:474-8.

15. Lan Lijuan L, Dafeng H, Shenghua, et al. Islet beta-cell function analysis of male HIV/AIDS patients who did not receive antiretroviral therapy. AIDS STDs in China. 2016;22:73-6.

16. AIDS Group, Society of Infectious Diseases,Chinese Medical Association. Third Edition of AIDS Diagnosis and Treatment Guidelines. (2015 Edition). Chinese Journal of Clinical Infectious Diseases2015;8: 385-401.

17. Joint Committee on the Development of Guidelines for the Prevention. and Control of blood lipidsin Chinese Adultãanguidelines for the prevention and treatment of dyslipidemia in Chinese adults 2016 revised edition. Chinses Circulation Journal. 2016;31(10):937-54.

18. Chinese Society of Endocrinology. Guidelines for the diagnosis and treatment of hyperuricemia and gout in China (2019). Chin J endocrinol Metab. 2020;36(1):1-13.

19. Chia-Jui Yang a,b, Hsiu-Yin Wang c, Tse-Chih Chou d,Chee-Jen Chang. Prevalence and related drug cost of comorbidities in HIV-infected patients receiving highly active antiretroviral therapy in Taiwan: A cross-sectional study.J Microbiol Immunol Infect. 2019; 52(5):720-727.

20. Geffner ME, Patel K, Miller TL, Hazra R, Silio M, Van Dyke RB, et al. Factors associated with insulin resistance among children and adolescents perinatally infected with HIV-1 in the pediatric HIV/AIDS cohort study. Horm Res Paediatr. 2011;76:386-91.

21. Sharma TS, Jacobson DL, Anderson L, Gerschenson M, Van Dyke RB, et al. Short communication: The relationship between mitochondrial dysfunction and insulin resistance in HIV-infected children receiving antiretroviral therapy. AIDS Res Hum Retroviruses. 2013;29:1211-7.

22. Idiculla J, Ravindra'n GD, D'Souza J, Singh G, Furruqh S. Diabetes mellitus, insulin resistance, and metabolic syndrome in HIV-positive patients in South India. Int J Gen Med. 2011;4:73-8.

23. Alvarez C, Salazar R, Galindez J, et al. Metabolic syndrome in HIV-infected patients receiving ARV therapy in Latin America. Braz J Infect Dis. 2010;14:256-63.

24. Mayanja BN, Kasamba I, Levin J, Namakoola I, Kazooba P, et al. COHORT PROFILE: The Complications of Long-Term Antiretroviral Therapy study in Uganda (CoLTART), a prospective clinical 
cohort. AIDS Res Ther. 2017;14:26.

25. Nazisa Hejazi1, Roslee Rajikan1. Choong CLeeK, et al. Metabolic abnormalities in adult HIV infected population on antiretroviral medication in Malaysia: a cross-sectional survey.BMC Public Health 2013;13:758-69.

26. Ataro Z, Ashenafi W, Fayera J, Abdosh T. Magnitude and associated factors of diabetes mellitus and hypertension among adult HIV-positive individuals receiving highly active antiretroviral therapy at Jugal Hospital, Harar, Ethiopia.HIV AIDS (Auckl). 2018;10:181-192.

27. Oscar HDivala, Amberbir A, Ismail Z, et al. The burden of hypertension, diabetes mellitus, and cardiovascular risk factors among adult Malawians in HIV care: consequences for integrated services. BMC Public Health. 2016;16:1243-54.

28. Han WM, Jiamsakul A, Kiertiburanakul S, Ng OT, Sim BL, et al. Diabetes mellitus burden among people living with HIV from the Asia-Pacific region. J Int AIDS Soc. 2019;22:e25236.

29. Wand H, Calmy A, Carey DL, Samaras K, Carr A, Law MG. on behalf of the INITIO Trial International Coordinating Committee, et al. Metabolic syndrome, cardiovascular disease and type 2 diabetes mellitus after initiation of antiretroviral therapy in HIV infection. AIDS2007;21:2445-53.

30. Karamchand S, Leisegang R, Schomaker M, Maartens G, Walters L, et al. Risk Factors for Incident Diabetes in a Cohort Taking First-Line Nonnucleoside Reverse Transcriptase Inhibitor-Based Antiretroviral Therapy. Medicine. 2016;95:e2844.

31. Nansseu JR, Bigna JJ, Kaze AD, Noubiap JJ. Incidence and Risk Factors for Prediabetes and Diabetes Mellitus Among HIV-infected Adults on Antiretroviral Therapy: A Systematic Review and Meta-analysis.Epidemiology2018;29:431-441.

32. Adelzon A, Paula M, Schechter SH, Tuboi, et al. Continuous Increase of Cardiovascular Diseases, Diabetes, and Non-HIV Related Cancers as Causes of Death in HIV-Infected Individuals in Brazil: An Analysis of Nationwide Data. Plos. 2014;9:1-5.

33. Takemoto JK, Miller TL, Wang J, Jacobson DL, Geffner ME, et al.Insulin resistance in HIV-infected youth is associated with decreased mitochondrial respiration.AIDS 2017;31:15-23.

34. Boccara F, Kumar P, Caramelli B, Calmy A, López JAG, et al. Evolocumab treatment in patients with HIV and hypercholesterolemia/mixed dyslipidemia: BEIJERINCK study design and baseline characteristics. Am Heart J. 2020;220:203-12.

\section{Figures}



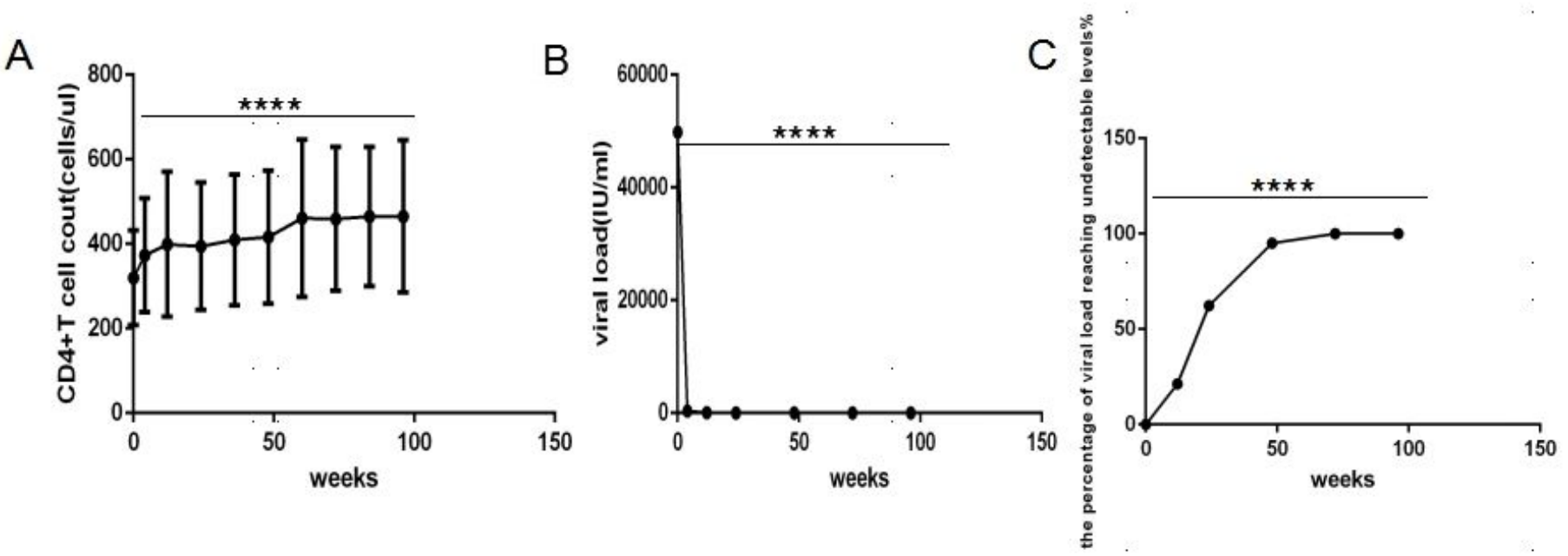

Figure 1

Effectiveness of HAART with TDF plus 3TC plus EFV primary treatment for human immunodeficiency virus infected male patients $(n=61)$. ONE ANOVA was used to compare CD4+ T cell count, viral load and the percentage of viral load reaching undetectable levels between different time point. A. CD4+ T cell count. B. viral load. C. the percentage of viral load reaching undetectable levels. Abbreviations: TDF, Tenofovir. 3TC, Lamivudine. EFV, Efavirenz. ${ }^{\star \star \star \star} \mathrm{P}<0.0001$. 

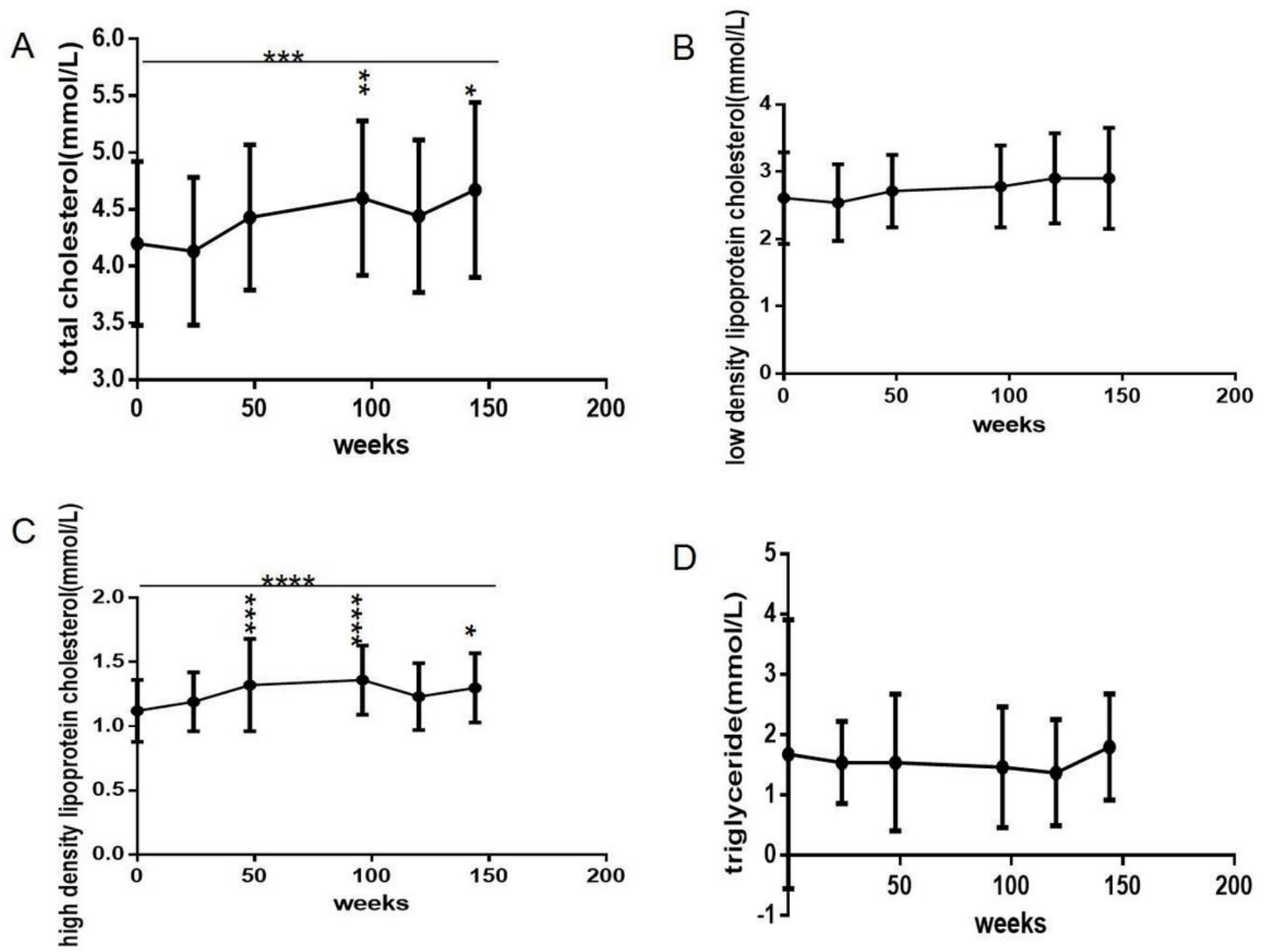

Figure 2

Long-term dynamic changes of lipid metabolic parameters within 144 weeks after initial highly active antiretroviral therapy with TDF plus 3TC plus EFV in treatment-naive HIV infected patients $(n=61)$. ANOVA was used to compare lipid metabolism parameters from baseline to 144 weeks and a paired t-test was used to compare lipid metabolism parameters between baseline and some follow-up time point. A. TC level. B. LDL-c level. C. HDL-c level. D. TG level.Abbreviations: TDF,Tenofovir. 3TC, Lamivudine. EFV, Efavirenz. TC, total cholesterol. LDL-c, low density lipoprotein cholesterol. HDL-c, high density lipoprotein cholesterol.TG, triglyceride. Comparison between different time points and compared with baseline,

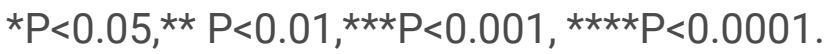



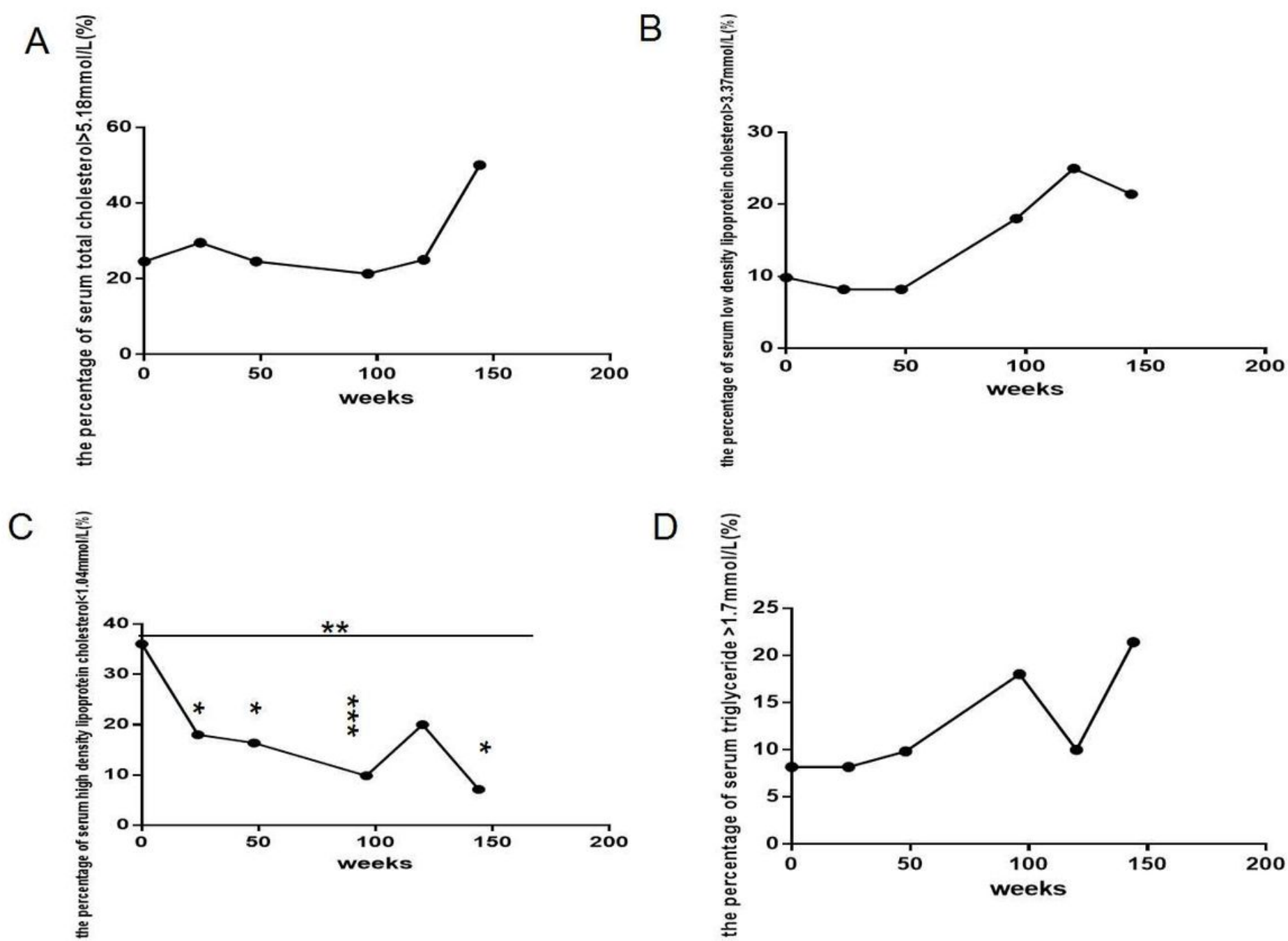

\section{Figure 3}

Long-term dynamic changes of the percentage of dyslipidemia within 144 weeks after initial highly active antiretroviral therapy with TDF plus 3TC plus EFV in treatment-naive HIV infected patients $(n=61)$. KruskalWallis $\mathrm{H}(\mathrm{K})$ test for $\mathrm{K}$ independent samples was used to compare the percentage of dyslipidemia from baseline to 144 weeks. Mann-Whitney test for two independent samples was used to compare the percentage of dyslipidemia between baseline and follow-up time point. A. the percentage of hypercholesterolemia $(\mathrm{TC}>5.18 \mathrm{mmol} / \mathrm{L})$. B. the percentage of hyper low density lipoprotein cholesterolemia ( $\mathrm{LDL}-\mathrm{c}>3.37 \mathrm{mmol} / \mathrm{L})$. C. the percentage of hypo high density lipoprotein cholesterolemia $(\mathrm{HDL}-\mathrm{C}<1.04 \mathrm{mmol} / \mathrm{L})$. D. the percentage of hypertriglyceridemia $(T \mathrm{G}>1.7 \mathrm{mmol} / \mathrm{L})$. Abbreviations:

TDF,Tenofovir. 3TC, Lamivudine. EFV, Efavirenz. TC, total cholesterol. LDL-C, low density lipoprotein cholesterol. HDL-c, high density lipoprotein cholesterol.TG, triglyceride. Comparison between different time points and compared with baseline, ${ }^{*} P<0.05$, ${ }^{\star \star} P<0.01,{ }^{\star * *} P<0.001$. 

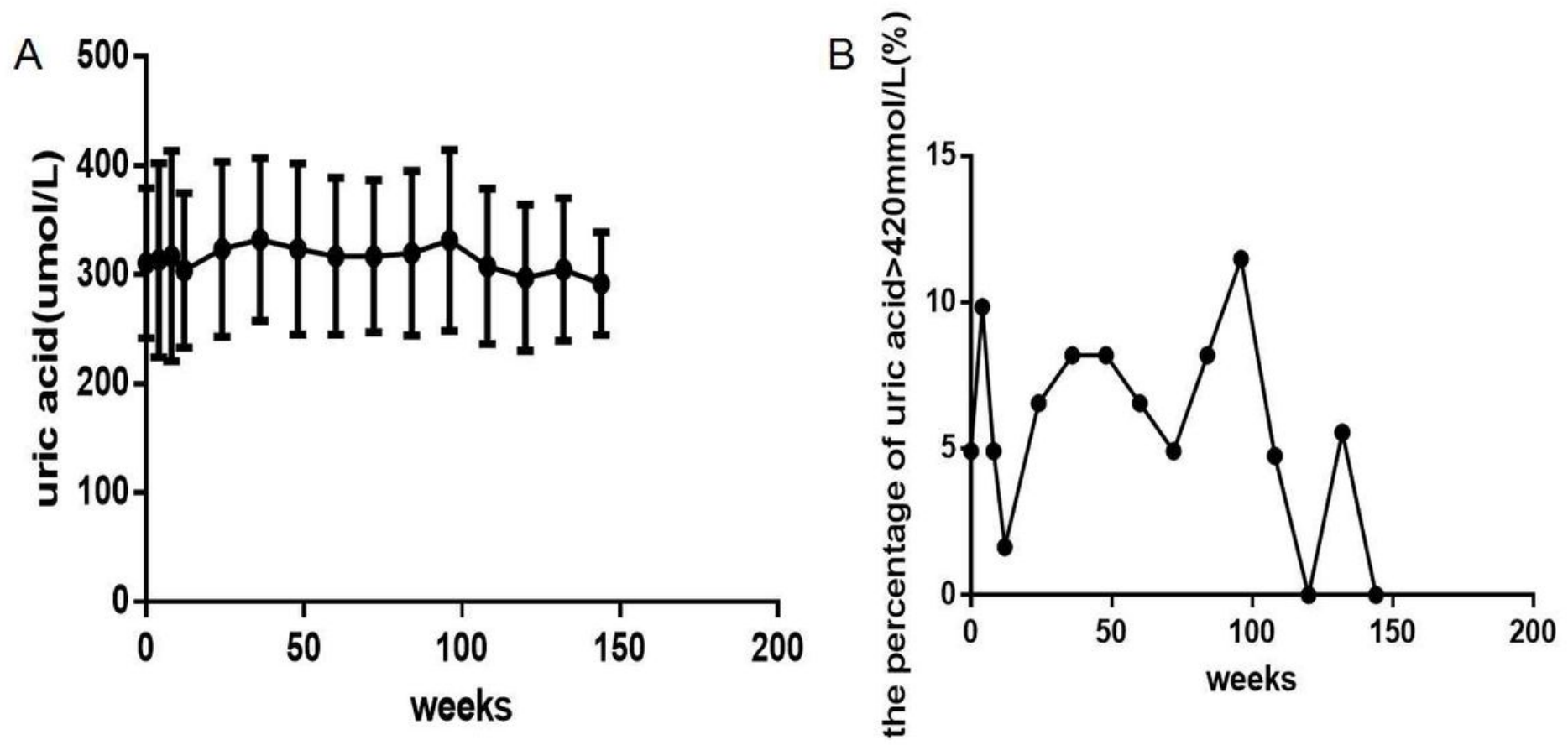

Figure 4

Long-term dynamic changes of UA and the percentage of hyperuricemia within 144 weeks after initial highly active antiretroviral therapy with TDF plus 3TC plus EFV in treatment-naive HIV infected patients $(n=61)$. ANOVA was used to compare UA level from baseline to 144 weeks and a paired $t$-test was used to compare UA level between baseline and some follow-up time point. Kruskal-Wallis $\mathrm{H}(\mathrm{K})$ test for $\mathrm{K}$ independent samples was used to compare the percentage of hyperuricemia from baseline to 144 weeks. Mann-Whitney test for two independent samples was used to compare the percentage of hyperuricemia between baseline and follow-up time point. A.UA level. B. the percentage of hyperuricemia.Abbreviations:TDF,Tenofovir. 3TC, Lamivudine. EFV, Efavirenz. UA, uric acid.Comparison between different time points and compared with baseline, all $P>0.05$. 

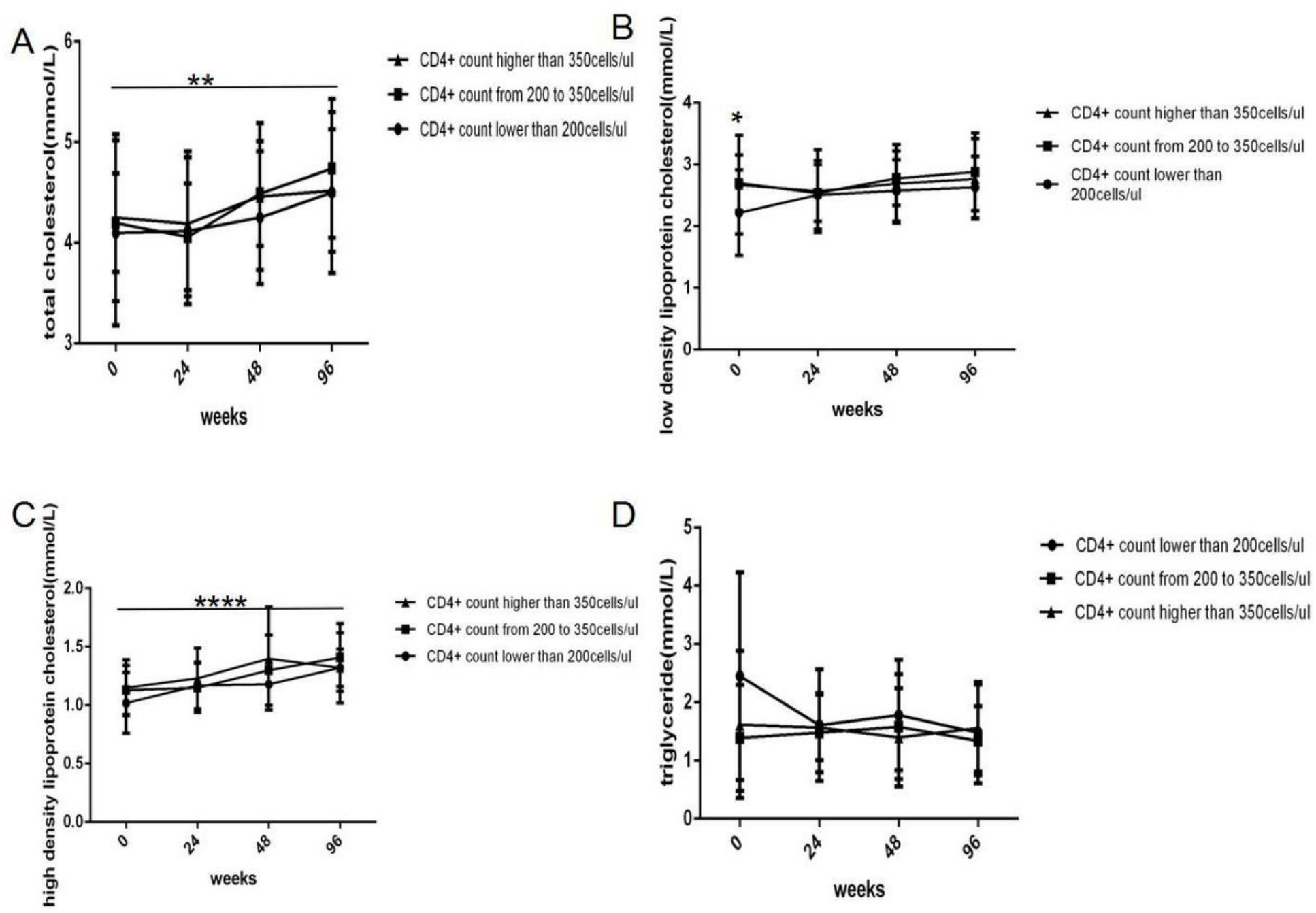

Figure 5

Long-term effect of baseline CD4+ T cell count on lipid metabolism parameters within 96 weeks after initial highly active antiretroviral therapy with TDF plus 3TC plus EFV in treatment-naive HIV infected patients $(n=61 ; 26,12,23$ cases in $<200$, from 200 to 350 , $>350$ groups, respectively). ONE ANOVA was used to compare lipid metabolism parameters between three groups at same time point and TWO ANOVA was used to compare lipid metabolism parameters between three groups from baseline to 96 weeks. A. TC level. B. LDL-c level. C. HDL-c level. D. TG level. Abbreviations: TDF, Tenofovir. 3TC, Lamivudine. EFV, Efavirenz. TC, total cholesterol. LDL-c, low density lipoprotein cholesterol. HDL-c, high density lipoprotein cholesterol.TG, triglyceride. Comparison between different time points and different CD4+ T cell count groups, $* P<0.05, * \star P<0.01, * \star \star \star P<<0.0001$. 


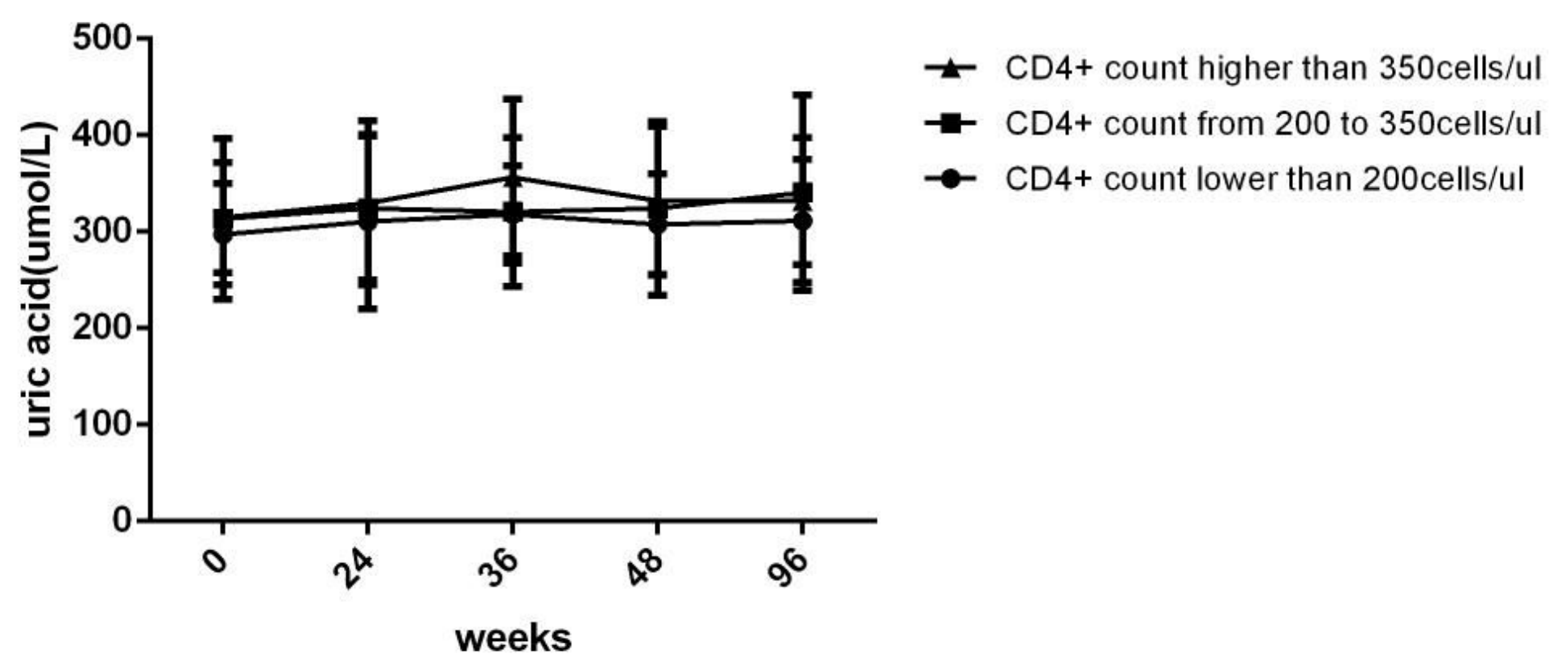

Figure 6

Long-term effect of baseline CD4+ T cell count on UA level within 96 weeks after initial highly active antiretroviral therapy with TDF plus 3TC plus EFV in treatment-naive HIV infected patients $(n=61 ; 26,12,23$ cases in<200, from 200 to 350, >350 groups, respectively). ONE ANOVA was used to compare UA between three groups at same time point and TWO ANOVA was used to compare UA between three groups from baseline to 96 weeks. Abbreviations: TDF, Tenofovir. 3TC, Lamivudine. EFV, Efavirenz. UA, uric acid. Comparison between different time points and different CD4+ T cell count groups, all $P>0.05$. 